\title{
HIV/AIDS among young women in Malawi: A review of risk factors and interventions
}

\author{
McDonald Nyalapa \& Cath Conn
}

\begin{abstract}
Young women aged 10-24 years in Malawi currently experience HIV prevalence of about $5 \%$. This high HIV prevalence amongst young women reflects a heart-breaking feature of the serious epidemic in southern Africa for the past five years. Given the serious situation it is vital to understand the risk factors faced by young women of Malawi, and further understanding of the interventions necessary to address the problem. A narrative review set out to explore the literature, retrieved from institutional reports and peer-reviewed publications, on the factors increasing young women's vulnerability to HIV in Malawi, and on interventions aimed at reducing their risk. Young women in Malawi are particularly vulnerable to HIV infection as a result of poverty, harmful gender norms, and economic and social inequities. Whilst there are some interventions in place, in the face of such a disproportionately difficult social and socio-economic environment, lack of resources and other systemic gaps, these are not sufficiently effective. Given the scale of the problem and the difficult environment experienced by young women, effective HIV prevention interventions remain critical. Further research is required to establish appropriate and effective interventions, and to address the social determinants of health, especially in relation to gender and rights.
\end{abstract}

Keywords: HIV risk factors, HIV interventions, girls, adolescents, young women, Malawi, Africa

This review is based on a presentation delivered by the first author at the Fourth Annual Interscholastic Student HIV Research Symposium on 29th June, 2017 in Auckland, New Zealand. The authors are from the School of Public Health \& Psychosocial Studies, Auckland University of Technology, Aotearoa New Zealand. For correspondence: mnyalapa@kcn.unima.mw 


\section{Introduction}

The HIV/AIDS epidemic continues to impose both a significant socio-economic and public health burden on low- and middle-income countries. Globally, there are about 36.9 million people living with HIV (UNAIDS, 2019). Despite constituting only $11 \%$ of the adult population, young women, aged 10-24 years, are particularly at high risk of HIV infection, accounting for $20 \%$ of new HIV infections among adults globally (UNAIDS, 2016). In sub-Saharan Africa, young women account for over $26 \%$ of new HIV infections among adults (UNAIDS, 2019). Southern Africa remains the region hardest hit by the HIV epidemic, with sexual behaviour largely determined by cultural and socio-economic contexts. In Malawi, factors that heighten HIV risk among young women include harmful gender norms and inequalities, insufficient access to education and sexual and reproductive health services, poverty and violence (National AIDS Commission [NAC], 2014a).

Although much research has been conducted on HIV/AIDS in the sub-Saharan African region, no specific review has been undertaken to analyse the risk factors and interventions relating to HIV among young women in Malawi. The review reveals that HIV risk among young women is shaped by socio-economic and cultural factors. Also, there are HIV prevention interventions in place, some of which are tried and tested in a variety of setting, but there is limited attention to addressing social determinants of health. Further research is required to investigate how current HIV prevention might incorporate gender and human rights elements and other social determinants of health in the Malawi context.

\section{Methods}

Literature for this review was retrieved and compiled from searches of computerised databases (ProQuest, MEDLINE, Scopus, CINAHL Plus and SPORTDiscus). The initial search strategy used a combination of key terms, "HIV" "AIDS" "girls" "women" "Africa" and "sub-Sahara" to identify potential articles. The search resulted in over 1,500 database hits. From this a further selection narrowed down to articles published in English from 1989 to 2017, relevant to HIV/AIDS and women. A final refining was conducted using "Malawi" as the context and this yielded 134 articles with a total of 28 articles meeting the inclusion criteria. In addition, Web-based sources and agency publications and reports were used in the final review for the background to the issue of HIV/AIDS in sub-Saharan Africa, and young women of Malawi.

\section{HIV/AIDS and young women in sub-Saharan Africa}

Sub-Saharan Africa, the region hardest hit by HIV epidemic, is home to over 24 million people living with HIV (UNAIDS, 2016). Eastern and southern Africa remain the epicentre of the global HIV epidemic, bearing almost $40 \%$ of the global burden (Dellar, Dlamini, \& Karim, 2015). While the general population prevalence of HIV/AIDS is very high in eastern and southern Africa (UNAIDS, 2019), the epidemic is unevenly distributed across the population, with some sub-populations being persistently more vulnerable than others (Dellar et al., 2015). Young women in eastern and southern Africa are an example of a sub-population which is disproportionately affected by HIV.

Young women's disproportionate risk of HIV has long been evident (Harrison, Colvin, Kuo, Swartz, \& Lurie, 2015). Across eastern and southern Africa, young women continue to be significantly more likely than their male counterparts to be infected 
by HIV (Harrison et al., 2015; UNAIDS, 2019). HIV prevalence in the general population across sub-Saharan Africa has decreased substantially yet the incidence of HIV infection remains high among young women (Dellar et al., 2015). Young women in eastern and southern Africa, epitomize a key population with levels of HIV infection comparable to the world's highly vulnerable populations, such as male and female sex workers (Beyrer et al., 2014).

Malawi is among those countries worst affected by the HIV epidemic in eastern and southern Africa. Malawi's adult HIV prevalence is at $10.8 \%$. This is in the context of being ranked one of the poorest countries in the world by GDP per capita (Kim et al., 2017). As a result of poverty, as well as gender inequalities and harmful cultural norms, the prevalence of HIV among young women is almost five times greater than young male counterparts, representing a massive health disparity (National Statistical Office [NSO] \& ICF, 2017). The reasons behind this disparity are discussed below.

\section{Factors influencing young women's vulnerability to HIV}

A wide range of factors are considered to escalate women's HIV risk globally. Factors such as gender power imbalances, poverty, sexual and reproductive health risk and socio-cultural factors have been shown to be prominent in increasing the risk of HIV among young women in Malawi.

The gendered context of HIV risk

Disproportionate and high HIV prevalence among young women in southern Africa is consistent with ways in which gender relations are structured (Jewkes \& Morrell, 2010). It reflects the unequal gender relations evident in young women's sexual partnerships with older men, in limited negotiating power within relationships and households, and in the rising advent of gender-based violence occurring in southern Africa (Dellar et al., 2015). Young women are more often victims of non-fatal violence, such as domestic abuse and sexual assault (Harrison et al., 2015). This exacerbates their vulnerability to HIV.

Poverty also pushes young women into age-disparate relationships, a key driver of HIV risk among young women (UNAIDS, 2015). For instance, young women may enter into relationships with older men in search of opportunities or material goods. These relationships disempower young women and expose them to unsafe sex, low condom use and increased contact with sexually transmitted infections (UNAIDS, 2015). Hence, disproportionately high HIV rates among young women in southern Africa have been linked to their relationships with older-aged partners (LeclercMadlala, 2008). Similarly, countries with generalised epidemics but outside Southern Africa, such as Cameroon, Nigeria, Togo, Chad, Ivory Coast and Rwanda consider age-disparate relationships as a key driver of HIV (Harling, Newell, Tanser, Kawachi, Subramanian, \& Bärnighausen, 2014).

\section{Sexual and reproductive risk factors}

Pregnancy is linked to the risk of HIV among young women. In southern Africa, 35$40 \%$ of women have their first child by the age of 19 , and over $70 \%$ by the age of 25 , indicating a heightened HIV risk through early and unprotected sex (Harrison et al., 2015). Moreover, approximately two-thirds of pregnancies among young women under the age of 25 are reported as unplanned or unwanted (Dellar et al., 2015).

Unwanted pregnancies are closely associated with physical abuse in southern Africa (Christofides et al., 2014). The pervasive context of women's disempowerment also 
significantly contributes to the advent of unwanted teenage pregnancies, as women feel little-to-no ability to control their sexual or reproductive lives (Christofides et al., 2014). Poverty also pushes teenage girls into pregnancy, perceiving it as security for their future, especially if the father of the child provides economic support (Kaufman, de Wet \& Stadler, 2001). Across southern Africa, more than 40\% of pregnant women attending antenatal health services are HIV-infected (Harrison et al., 2015). Once pregnant, young women are also more likely to acquire HIV infection because concerns about their partners' HIV status are superseded by the reduced need for contraception (Peltzer, Jones, Weiss, Villar-Loubet, \& Shikwane, 2013).

Contraceptive methods are an important aspect of HIV prevention among young women. The use of barrier methods, such as condoms, significantly reduces young people's HIV risk (Dellar et al., 2015). However, contraceptive use is often low and inconsistent in southern Africa, with high rates of method discontinuation (Harrison et al., 2015). Given the context of unequal partnerships, it also implies that, women have little or no say on contraceptive choices, with men more likely to disapprove of contraceptive use (Harrison et al., 2015).

\section{Sociocultural factors}

Harmful cultural practices have been considered a key driver of HIV in most African countries, including in Malawi. A common example of such practices is the fisi, "the hyena" ritual. It refers to a man who sexually initiates girls to complete the rites into womanhood (Banda \& Kunkeyani, 2015). There are several types of fisi based on the cultural role he performs. Among these, there is a type of fisi whereby a girl who has just attained puberty has to have sex with a man "to remove dust", meant to cleanse and purify her after her first menstruation (Banda $\&$ Kunkeyani, 2015). This practice prevails in other Malawian tribes, such as sena despite calls by women's rights activists for its eradication.

The unfortunate reality of this fisi ritual is that the fisi can engage in sex with several girls on the same night and without any protection, thereby heightening the vulnerability of girls to sexually transmitted infections including HIV (Munthali, Chimbiri, \& Zulu, 2004). Girl rights' campaigners have termed this practice "statutory rape" which violates girls' and women's human rights and dignity in the name of culture (Kamlongera, 2007).

Research shows that the fisi ritual has not died out completely but it has undergone modification (Banda \& Kunkeyani, 2017). It is now common for the fisi to carry a "shield", a brand name of a condom, currently used to mean any condom in Malawi. Alternatively, the fisi goes for a mandatory HIV test before being assigned any sexual roles. Although this signifies a step in incorporating safer sex in cultural practices, consistency of such prevention strategies is largely unknown.

Another example of harmful cultural practices that significantly heighten the risk of HIV among young women is chocolo, "wife inheritance" (Bisika, 2008). This is a practice whereby the wife of a deceased husband is "inherited" by his brother, cousin or nephew (Banda \& Kunkeyani, 2015). In this practice, the woman is forced to marry the deceased husband's close relation, thus taking advantage of the women's lack of power in decision making. Much of the time, HIV testing does not take place, as this is seen as a cultural arrangement. This practice has significantly contributed to the spread of HIV in Malawi. Although this practice is in general decline, it is difficult to conclude that it has completely died out as isolated cases of such acts are still recorded in Malawi (Banda \& Kunkeyani, 2015).

The chokolo practice has also seen modifications following calls by rights' campaigners to abolish it. Recent research shows that in some cases, before the woman is inherited by the surviving relative, both parties have to undergo an HIV 
test (Banda \& Kunkeyani, 2015). If one of the parties is found positive, the whole process is discontinued. In some Malawian communities, the widow is not remarried but stays within the compound and is looked after materially by the relatives of her deceased husband (Banda $\&$ Kunkeyani, 2015). As long as there is no sexual contact, the woman's HIV risk is reduced.

Other practices, such as early marriages for girls, are also on the decline in Malawi. This is because of advocacy programmes by the government and other nongovernmental organizations (NGOs), such as World Vision, Girl Guide and Youth Network Organization (Banda \& Kunkeyani, 2015). Moreover, traditional chiefs have been empowered to monitor implementation of laws against under-age marriages. This already shows the potential to reduce and eradicate early marriages. A typical example of such an iconic chief is Theresa Kachindamoto of Dedza District in central Malawi, who has singlehandedly terminated over 330 child marriages and has managed to send young girls back to school. She is popularly known as "the terminator of child marriages".

\section{HIV prevention interventions available for adolescent girls and young women}

\section{Abstinence, monogamy and condom use}

HIV prevention since the 1980s has been grounded in "ABC" approaches which encourage abstinence, adhering to one sexual partner (be faithful), and the use of condoms (Kerwin, Folley, Thornton, Basinga, \& Chinkhumba, 2011). People who adhere to $\mathrm{ABC}$ are presumed to have a lower risk of HIV (Kerwin, et al., 2011). However, women in Malawi and other African societies have limited control over their husbands' faithfulness due to lack of agency and voice in sexual matters (Baxter \& Abdool Karim, 2016). Moreover, abstinence-only programmes have shown little to no impact on the incidence of unprotected sex, number of partners and condom use (Underhill, Montgomery, \& Operario, 2007). Although the ABC approach has demonstrated some positive governance of behaviour among young people, it is largely incommensurate with existing socio-cultural formations and takes little heed of gender and economic inequities faced by young women (Reid-Hresko, 2014).

Condoms are a crucial aspect of HIV prevention programmes. If used correctly and consistently, condoms offer high levels of protection against HIV, other sexually transmitted infections and pregnancy (Holmes, Levine, \& Weaver, 2004). In Malawi, a low rate of $25.7 \%$ of women report using condoms with non-spousal regular partners (National Statistics Office \& ICF Macro, 2011). Chronic condom stock-outs at the facility level and public access areas remain a huge challenge for Malawi largely due to delays in procurement and lack of funding for healthcare (Dasgupta, Zaba, \& Crampin, 2015; NAC, 2014b). Such poor availability reduces the effectiveness of condoms as a prevention option, particularly for young women living in rural areas, who may not manage to purchase condoms from grocery stores due to economic deprivation and stigma.

Gender norms negatively affect condom use among young women in Malawi. Gender power imbalances often limit women's ability to negotiate condom use with their partners (Baxter \& Abdool Karim, 2016). Power imbalances in relationships result in gender-based violence which also heightens young women's HIV vulnerability (Abdool Karim, Sibeko, \& Baxter, 2010). A notable example of power imbalances in intimate relationships is vividly illustrated by Raghallaigh, Moton and Alen (2017), whereby a woman faces physical and sexual violence by questioning her husband's 
fidelity. In cases where women have no power over their own bodies, their risk for HIV infection is escalated (Raghallaigh et al., 2017). Given these power issues in sexual relationships, effective condom availability must be configured alongside interventions which empower women.

Previous research showed that condom use in Malawi was introduced against the background of strong objections, with the most important being the nexus of condom use and the type of relationship (Chimbiri, 2007). Over the last decade, the use of condoms was perceived to be associated with commercial sex work (Chimbiri, 2007). Men were found to believe that using a condom was a sign that the relationship is not exclusive, thereby terming a condom as an "intruder" in intimate relationships (Chimbiri, 2007). Moreover, condoms were found to reduce the pleasure of sex and this was a key factor for decreased condom use among men in Malawi (John, Babalola, \& Chipeta, 2015). However, sensitization campaigns on correct and consistent condom use currently underway in Malawi provide the hope to increase uptake of condoms by men (Haddad et al., 2018).

\section{Behaviour change interventions}

Various behavioural change interventions to prevent HIV have been implemented in Malawi. These include peer education, media communication, school-based HIV prevention education and behavioural counselling (Baxter \& Abdool Karim, 2016). Evidence suggests that school-based HIV prevention education increases young people's knowledge of HIV and mitigates risky practices associated with HIV (Butts et al., 2017; Fonner, Armstrong, Kennedy, O'Reilly, \& Sweat, 2014; Miedema, Maxwell, \& Aggleton, 2011).

Experts have suggested that in-school HIV prevention education can be a key intervention in reducing young people's risk for HIV (Okonofua, 2015; Wood \& Rolleri, 2014). However, there are persistent challenges in relation to the design and implementation of this intervention including in Malawi. These include lack of sexuality education skills among teachers (Francis \& DePalma, 2015); lack of consistency in sexuality education programmes (Fonner et al., 2014); lack of emphasis on vulnerable groups, such as young women (Miedema et al., 2011); and crucially the lack of consideration of the social determinants of health in a given context. Because of these enormous challenges, it can be concluded that sexuality education programmes need to be carefully developed taking into consideration context in order to reduce HIV risk among young women.

\section{HIV counselling and testing}

HIV counselling and testing is a low-cost, effective HIV prevention strategy (Sweat et al., 2000). However, global estimates indicate that about 52\% (9.4 million) of all people living with HIV do not know their status (UNAIDS, 2019). In Malawi, one million people living with HIV did not know their status in 2014, with those undiagnosed still proving a challenge (NAC, 2014a). Although HIV counselling and testing services are being scaled up, denial, stigma and a lack of understanding of risk and vulnerability result in low HIV testing rates (NAC, 2014a). In many African countries, discrimination and social marginalization are still experienced by people who are affected by HIV, thereby increasing their reluctance to seek testing (Karim, 2011). Recent research in Malawi has also demonstrated physical violence by men against their spouses for seeking HIV counselling and testing services (Lavender et al., 2019; Raghallaigh, 2017).

Ambitious global targets for HIV testing and treatment have been set, aiming to have 90\% of people living with HIV knowing their status, 90\% of people who know their status receiving treatment, and $90 \%$ of people on HIV treatment resulting in a suppressed viral load, by 2020 (UNAIDS, 2016). These targets are admirable, but 
require massive scale-up of HIV testing and innovative ways to reach people who are unaware of their status, including in Malawi. Given the social environment, facilitybased counselling and testing should be complemented with empowerment-based community or outreach programmes in order to increase uptake of such services among young women in Malawi. Empowerment-based HIV counselling and testing programmes need to confidentially serve the proportion of women who refuse an HIV test until they obtain approval from their husbands (Tenthani et al., 2015).

\section{Cash incentives for HIV prevention}

Cash transfers to young people, in order to incentivise safer behaviour, have recently emerged as a new strategy to reduce young people's vulnerability to HIV (Dellar et al., 2015). This strategy looks promising; an independent randomised controlled trial conducted in Malawi demonstrated that female high school students who received cash transfers were 64\% less likely to be HIV infected (Baird, Chirwa, McIntosh, \& Özler, 2010). The main reason behind the success of the cash transfer programme is its ability to address "upstream factors" that heighten the risk of HIV among women (Heise, Lutz, Ranganathan, \& Watts, 2013). Direct cash transfers reduce povertyrelated stress and decrease the probability that girls or women will resort to transactional sex for food or other goods (Heise et al., 2013).

In Malawi, the social cash transfer programme (SCTP) was established in 2006 with support from the Global Fund (Government of Malawi [GOM], 2015). The programme mainly targets women; now, the programme has 373, 000 beneficiaries (GOM, 2015). Over the years, funding for this programme has been increasing but now the contribution has decreased due to funding problems in the public sector (GOM, 2015). For example, the funding for SCTP has dropped from Malawi Kwacha (MK) 450 million (equivalent to US\$ 600,000) in 2014, to MK 350 million (equivalent of US\$ 450,000) in 2015 (GOM, 2015). This reduction in funding signals a challenge to the sustainability of the SCTP.

\section{Medical male circumcision}

Voluntary medical male circumcision (VMMC) has been demonstrated to reduce the risk of male-to-female HIV transmission by up to 61\% (Auvert et al., 2005; Bailey et al., 2007; Gray et al., 2007; Siegfried, Muller, Deeks, \& Volmink, 2009). VMMC is being implemented in different countries as a priority component of HIV prevention programmes, particularly in those countries with an increased burden of HIV, such as Malawi (UNAIDS, 2016). Unfortunately, VMMC is associated with low condom use in Malawi because men do not know that VMMC only partially protects against HIV (Carrasco \& Kaufman, 2017).

Currently, there is an ambitious global target of 20 million circumcisions $(80 \%$ coverage) by 2020 (UNAIDS, 2016). However, male circumcision as an intervention has its critics. As well as being problematic because it might provide a sense that being circumcised means that men do not need to use a condom, it is also perceived as intruding and disrupting the ethics (culture and religion) of people (Wamai, Morris, Bailey, Klausner, \& Boedicker, 2015). Current debates on male circumcision also centre on reduced sexual satisfaction and the adverse effects of circumcision (Wamai et al., 2015). As such, VMMC coverage is very low $(11.5 \%)$ and is fraught with mixed perceptions in Malawi (Parkhurst, Chilongozi, \& Hutchinson, 2015). There is a need to approach use of VMMC with caution, and sensitise the general public, particularly young men to ensure that any uptake is appropriately informed. Other innovative strategies, such as incentivising VMMC, might also be considered to increase uptake by young men. 


\section{Conclusion}

This review has identified harmful gender norms and inequities, and poverty as factors exacerbating young women's HIV risk in Malawi. HIV prevention is being driven by global programmes and targets, such as the Fast-Track (90-90-90 targets), and in response, Malawi is implementing community-based HIV prevention interventions. This review has summarised the main current interventions as they relate to young women. However, given the lack of specific research from the Malawi context, the review relied mostly on research from southern Africa. The literature is silent on the degree to which current HIV prevention interventions address gender and human rights issues in Malawi. Studies are required to establish the effectiveness of existing interventions, especially considering how gender and human rights can be addressed as part of this vital agenda.

\section{Acknowledgements}

This review was also supported by Dr Nadia Charania, senior lecturer at Auckland University of Technology, Auckland, New Zealand. 


\section{References}

Abdool Karim, Q., Sibeko, S., \& Baxter, C. (2010). Preventing HIV infection in women: A global health imperative. Clinical Infectious Diseases, 50(Supp 3), S122-S129. doi:10.1086/651483

Auvert, B., Taljaard, D., Rech, D., Lissouba, P., Singh, B., Bouscaillou, J., ... Lewis, D. (2013). Association of the ANRS-12126 Male Circumcision Project with HIV levels among men in a South African township: Evaluation of effectiveness using cross-sectional surveys. Public Library of Science Medicine, 10, e1001509. doi:0.1371/journal.pmed.1001509

Bailey, R. C., Moses, S., Parker, C. B., Agot, K., Maclean, I., Krieger, J. N., ... Ndinya-Achola, J.O. (2007). Male circumcision for HIV prevention in young men in Kisumu, Kenya: A randomised controlled trial. Lancet, 369, 643-646. Retrieved from: http://www.ncbi.nlm.nih.gov/pubmed/17321310

Baird, S., Chirwa, E., McIntosh, C., \& Özler, B. (2010). The short-term impacts of a schooling conditional cash transfer program on sexual behavior of young women. Health Economics, 19(S1), 55-68. doi:10.1002/hec.1569

Banda, F., \& Kunkeyani, T. E. (2015). Renegotiating cultural practices as a result of HIV in the eastern region of Malawi. Culture, Health \& Sexuality, 17(1), 3447. doi:10.1080/13691058.2014.944569

Baxter, C., \& Abdool Karim, S. (2016). Combination HIV prevention options for young women in Africa. African Journal of AIDS Research, 15(2), 109-121. doi: 10.2989/16085906.2016.1196224

Beyrer, C., Baral, S. D., Weir, B., Curran, J. W., Chaisson, R. E., \& Sullivan, P. (2014). A call to action for concentrated HIV epidemics. Current opinion in HIV and AIDS, 9(2), 95-100. doi:10.1097/COH.0000000000000043

Bisika, T. (2008). Cultural factors that affect sexual and reproductive health in Malawi. Journal of Family Planning and Reproductive Healthcare, 34(2), 7980. doi:10.1783/jfp.34.2.79

Butts, S. A., Kayukwa, A., Langlie, J., Rodriguez, V. J., Alcaide, M. L., \& Chitalu, N. (2017). HIV knowledge and risk among Zambian adolescent and younger adolescent girls: Challenges and solutions. Sex Education, 1-13. doi: $10.1080 / 14681811.2017 .1370368$

Carrasco, M. A. \& Kaufman, M. R. (2017). Correlates of condom use and procedure knowledge among men accessing voluntary medical male circumcision in Malawi. International Journal of Behavioral Medicine, 24(2), 305-311. doi: $10.1007 / \mathrm{s} 12529-016-9594-3$

Chimbiri, A. M. (2007). The condom is an "intruder" in marriage: Evidence from rural Malawi. Social Science and Medicine, 64, 1102-1115. doi: 10.1016/j.socscimed.2006.10.012

Christofides, N. J., Jewkes, R. K., Dunkle, K. L., McCarty, F., Shai, N. J., Nduna, M., \& Sterk, C. (2014). Risk factors for unplanned and unwanted teenage pregnancies occurring over two years of follow-up among a cohort of young South African women. Global Health Action, 7. doi:10.3402/gha.v7.23719

Dasgupta, A. Z., Zaba, A., \& Crampin, A. C. (2015). Contraceptive dynamics in rural northern Malawi: A prospective longitudinal study. International Perspectives on Sexual and Reproductive Health, 41 (3), 145-154. doi: 10.1363/4114515

Dellar, R. C., Dlamini, S., \& Karim, Q. A. (2015). Adolescent girls and young women: Key populations for HIV epidemic control. Journal of the International AIDS Society, 18(2 Suppl 1), 64-70. doi: 10.7448/IAS.18.2.19408 
Fonner, V. A., Armstrong, K. S., Kennedy, C. E., O'Reilly, K. R., \& Sweat, M. D. (2014). School based sex education and HIV prevention in low- and middleincome countries: A systematic review and meta-analysis. PLoS One, 9(3), e89692. doi: 10.1371/journal.pone.0089692

Francis, D. A. \& DePalma, R. (2015). 'You need to have some guts to teach': Teacher preparation and characteristics for the teaching of sexuality and HIV/AIDS education in South African schools. SAHARA J, 12, 30-38. doi: 10.1080/17290376.2015.1085892

Government of Malawi. (2015). Malawi AIDS response progress report 2015. Retrieved from http://www.unaids.org/sites/country/MWI_narrative_report_2015

Gray, R. H., Kigozi, G., Serwadda, D., Makumbi, F., Watya, S., Nalugoda, F., ... Wawer, M. J. (2007). Male circumcision for HIV prevention in men in Rakai, Uganda: A randomised trial. Lancet, 369(9562), 657-666. doi:10.1016/S0140-6736(07)60313-4

Haddad, L. B., Tang, J. H., Krashin, J., Ng'ambi, W., Tweya, H., Samala, B., ... Phiri, S. (2018). Factors associated with condom use among men and women living with HIV in Lilongwe, Malawi: A cross-sectional study. BMJ Sexual Reproductive Health 44(1), 1-12. doi: 10.1136/bmjsrh-2017-101825

Harling, G., Newell, M.-L., Tanser, F., Kawachi, I., Subramanian, S., \& Bärnighausen, T. (2014). Do age-disparate relationships drive HIV incidence in young women? Evidence from a population cohort in rural KwaZuluNatal, South Africa. Journal of Acquired Immune Deficiency Syndromes 66(4), 443-451. doi:10.1097/QAI.0000000000000198

Harrison, A., Colvin, C. J., Kuo, C., Swartz, A., \& Lurie, M. (2015). Sustained high HIV incidence in young women in Southern Africa: Social, behavioral, and structural factors and emerging intervention approaches. Current HIV/AIDS Reports, 12(2), 207-215. doi:10.1007/s 11904-015-0261-0

Heise, L., Lutz, B., Ranganathan, M., \& Watts, C. (2013). Cash transfers for HIV prevention: Considering their potential. Journal of International AIDS Society, 16 (1), 18615. doi:10.7448/IAS.16.1.18615

Holmes, K. K., Levine, R., \& Weaver, M. (2004). Effectiveness of condoms in prenting sexually transmitted infections. Bulletin of the World Health Organization, 82(6), 454-461. doi:10.1590/S0042-96862004000600012

Jewkes, R. \& Morrell, R. (2010). Gender and sexuality: Emerging perspectives from the heterosexual epidemic in South Africa and implications for HIV risk and prevention. Journal of the International AIDS Society, 13(1) 1. doi: 10.1186/1758-2652-13-6

John, N. A., Babalola, S., \& Chipeta, E. (2015). Sexual pleasure, partner dynamics and contraceptive use in Malawi. International Perspectives on Sexual and Reproductive Health, 41 (2), 99-107. doi:10.1363/4109915

Kamlongera, A. (2007). What becomes of her: A look at the Malawian fisi culture and its effects on young girls. Agenda, 21(74), 81-87. doi: $10.1080 / 10130950.2007 .9674879$

Karim, S. S. A. (2011). Stigma impedes AIDS prevention. Nature, 474(7349), 29-31. doi: $10.1038 / 474029 a$

Kaufman, C. E., de Wet, T.,\& Stadler, J. (2001), Adolescent Pregnancy and Parenthood in South Africa. Studies in Family Planning, 32: 147-160. doi: 10.1111/j.1728-4465.2001.00147.x

Kerwin, J. T., Folley, S. M., Thornton, R. L., Basinga, P., \& Chinkhumba, J. (2011). Missing safer sex strategies in HIV prevention: A call for further research. African Population Studies, 25(2), 286-297. Retrieved from http://aps.journals.ac.za 
Kim, M. H., Mazenga, A. C., Ahmed, S., Paul, M. E., Kazembe, P. N., \& Abrams, J. E. (2017). High self-reported non-adherence to anti-retroviral therapy amongst adolescents living with HIV in Malawi: Barriers and associated factors. Journal of the International AIDS Society, 2O(1), 21437. doi: 10.7448/IAS.20.1.21437

Lavender, T., Wakasiaka, S., Chimwaza, A., Wood, R., Omoni, G., Mukhwana, R., ... Edozien, L. (2019). A qualitative study of partner engagement in HIV testing in Malawi and Kenya. Culture, Health \& Sexuality, 3(4), 1-15. doi.org/10.1080/13691058.2018.1542509

Leclerc-Madlala, S. (2008). Age-disperate and intergenerational sex in the southern Africa: The dynamics of hypervulnerability. Aids, 22, S17-S25. doi: 10.1097/01.aids.0000341774.86500.53

Miedema, E. A., Maxwell, C., \& Aggleton, P. (2011). Education about HIV/AIDS-theoretical underpinnings for a practical response. Health Education Research, 26(3), 516-525. doi:10.1093/her/cyq088

Munthali, A. C., Chimbiri, A., \& Zulu, E. (2004). Adolescent and reproductive health in Malawi: A synthesis of research evidence. Retrieved from http://www.ndr.mw.8080/xmlui/bitstream/handle/123456789/254

Narasimhan, M., Askew, I., \& Vermund, S. H. (2016). Advancing sexual and reproductive health and rights of young women at risk of HIV. The Lancet Global Health, 4(10), e684-e685. doi:10.1016/S2214-109X(16)30151-6

National AIDS Commission. (2014a). National HIV prevention strategy 2015-2020. Lilongwe, Malawi: Author.

National AIDS Commission. (2014b). 2015-2020 national strategic plan for HIV and AIDS. Lilongwe, Malawi: Author.

National Statistics Office \& ICF Macro. (2011). Malawi Demographic Health Survey 2010. Zomba, Malawi: NSO and ICF Macro.

National Statistical Office \& ICF. (2017). Malawi Demographic Health Survey 201516. Zomba, Malawi: NSO and ICF.

Okonofua, F. (2015). Assessing the impact of sexuality and HIV/AIDS education in Africa. African Journal of Reproductive Health, 19(2), 9-10. Retrieved from http://search.proquest.com/docview/1707560851?accountid=8440

Parkhurst, J. O., Chilongozi, D., \& Hutchinson, E. (2015). Doubt, defiance, and identity: Understanding resistance to male circumcision for HIV prevention in Malawi. Social Science and Medicine, 135, 15-22. doi: 10.1016/j.socscimed.2015.04.020

Peltzer, K., Jones, D., Weiss, S. M., Villar-Loubet, O., \& Shikhwane, E. (2013). Sexual risk, serostatus and intimate partner violence among couples during pregnancy in rural South Africa. AIDS and Behaviour, 17, 508-516. doi: $10.1007 / \mathrm{s} 10461-012-0185-2$

Raghallaigh, M., Morton, S., \& Allen, M. (2017). HIV transmission as a form of gender-based violence: Experiences of women in Tigray, Ethiopia. International Social Work, 60(4), 941-953. doi:10.1177/0020872815594224

Reid-Hresko, J. (2014). Our bodies are our own: Resistance to ABC-based HIVprevention programmes in northern Tanzanian conservation organisations. Culthure, Health and Sexuality, 16(7), 765-779. doi: / 10.1080/13691058.2014.911959

Siegfried, N., Muller, M., Deeks, J. J., \& Volmink, J. (2009). Male circumcision for prevention of heterosexual acquisition of HIV in men. The Cochrane Database of Systematic Reviews, 15(2). doi: 10.1002/ 14651858.CD003362.pub2 
Sweat, M., Gregorich, S., Sangiwa, G., Furlonge, C., Balmer, D., Kamenga, C., . . . Coates, T. (2000). Cost-effectiveness of voluntary HIV-1 counselling and testing in reducing sexual transmission of HIV-1 in Kenya and Tanzania. The Lancet, 356(9224), 113-121. doi:10.1016/S0140-6736(00)02447-8

Tenthani, L., Haas, A. D., Egger, M., van Oosterhout, J. J., Jahn, A., Chimbwandira, F., ... Keiser, O. (2015). HIV testing among pregnant women who attend antenatal care in Malawi. Journal of Acquired Immune Deficiency Syndromes, 69(5), 610-614. doi:10.1097/QAI.0000000000000669

UNAIDS. (2015). Empower young women and adolescent girls: Fast-tracking the end of the AIDS epidemic in Africa. Retrieved from http://www.unaids.org/sites/default/files/media_asset/JC2746_en.pdf

UNAIDS. (2016). Global AIDS update 2016. Retrieved from http://www.who.int/hiv/pub/arv/global-aids-update-2016-pub

UNAIDS. (2019). Global HIV \& AIDS statistics - 2018 fact sheet. Retrieved from http://www.unaids.org/en/resources/fact-sheet

Underhill, K., Montgomery, P., \& Operario, D. (2007). Sexual abstinence only programmes to prevent HIV infection in high income countries: systematic review. British Medical Journal, 335(7613), 248. Retrieved from http://www.bmj.com/content/335/7613/248.abstract

Wamai, R. G., Morris, B. J., Bailey, R. C., Klausner, J. D., \& Boedicker, M. N. (2015). Male circumcision for protection against HIV infection in subSaharan Africa: The evidence in favour justifies the implementation now in progress. Global Public Health, 10(5-6), 639-666. doi: $10.1080 / 17441692.2014 .989532$

Wood, L., \& Rolleri, L. A. (2014). Designing an effective sexuality education curriculum for schools: Lessons gleaned from South(ern) African literature. Sex Education, 14(5), 525-542. doi:10.1080/14681811.2014.918540 